\title{
Conductance fluctuations at the quantum Hall plateau transition
}

\author{
F. Hohls, * U. Zeitler, and R. J. Haug \\ Institut für Festkörperphysik, Universität Hannover, Appelstrasse 2, D-30167 Hannover, Germany
}

(Received 18 April 2002; published 15 August 2002)

\begin{abstract}
We analyze the conductance fluctuations observed in the quantum Hall regime for a bulk two-dimensional electron system in a Corbino geometry. We find that characteristics like the power spectral density and the temperature dependence agree well with simple expectations for universal conductance fluctuations in metals, while the observed amplitude is reduced. In addition, the dephasing length $L_{\Phi} \propto T^{-1 / 2}$, which governs the temperature dependence of the fluctuations, is surprisingly different from the scaling length $L_{s c} \propto T^{-1}$ governing the width of the quantum Hall plateau transition.
\end{abstract}

DOI: 10.1103/PhysRevB.66.073304

PACS number(s): 73.23.-b, 73.43.-f

Conductance fluctuations are probably one of the most prominent features occurring in the mesoscopic world. For metallic (diffusive) systems at low enough temperature these so-called universal conductance fluctuations (UCF's) are characterized by an amplitude $\sim e^{2} / h$, independent on sample size and material, and they are generally believed to be well understood. ${ }^{1}$

Similar conductance fluctuations are also observed in mesoscopic quantum Hall (QH) systems, however, the underlying physics still remains an open question. Interpretations of different experiments cover modifications of UCF in high fields, ${ }^{2-4}$ tunneling between opposite edge states through bulk inhomogeneities, ${ }^{5-7}$ influences of charging effects, ${ }^{8,9}$ and networks of compressible-incompressible regions. ${ }^{10}$ Common to all the previous experiments is a sample geometry in the form of a (Hall) bar, the importance of edge channels, and a typical sample width $w$ of 1-3 $\mu \mathrm{m}$. Transport in edge channels and the coupling of both edges due to single impurities is strongly relevant, making the applicability of a general UCF theory problematic.

Here we will address the conductance fluctuations in the quantum Hall regime due to transport through the disordered bulk of a two-dimensional electron system (2DES). In order to avoid any edge effects we use a Corbino geometry. The large width $w=6 \mu \mathrm{m}$, considerably above the elastic scattering length $l_{\mathrm{el}}<1 \mu \mathrm{m}$, suppresses transport through individual impurities. Analyzing the temperature dependence of small fluctuations superimposed on the conductance peak of the $\mathrm{QH}$ plateau transition we find a behavior very common to UCF in metallic systems, however, with a considerably reduced absolute value of the UCF amplitude.

The samples used for this work are based on modulation doped GaAs/AlGaAs heterostructures. Additional impurities (Be or $\mathrm{Si}$ ) added into the 2DES (Refs. 11 and 12) yield an enhanced short-range scattering and a small elastic-scattering length. Throughout this paper we present data measured on a sample with an electron mobility $\mu=2 \mathrm{~m}^{2} / \mathrm{Vs}$, an electron density $n=2.1 \times 10^{15} \mathrm{~m}^{-2}$, and a density of Be impurities $n_{B e}=2 \times 10^{14} \mathrm{~m}^{-2}$. We confirmed our results with measurements on other samples, containing both $\mathrm{Si}$ and Be impurities, with mobilities ranging up to $\mu=12 \mathrm{~m}^{2} / \mathrm{Vs}$. Using annealed AuGe-Ni contacts the samples were patterned into Corbino geometry with a ring width $w=6 \mu \mathrm{m}$, and an inner radius $r=60 \mu \mathrm{m}$.
The samples were mounted on the cold finger of a dilution refrigerator with a base temperature $T<20 \mathrm{mK}$ and positioned into the center of a superconducting solenoid. We measured the current with a lock-in technique for fixed ac voltage $(9 \mathrm{~Hz})$ as a function of magnetic field and temperature. We carefully checked for heating effects by varying the amplitude of the excitation voltage at the same temperature. Excitation amplitudes adapted to the different temperature regimes were used to avoid heating at the lowest temperatures and still ensure high enough resolution of the fluctuations at elevated temperatures. The voltage was fixed to $1 \mu \mathrm{V}$ for $T<50 \mathrm{mK}, 2 \mu \mathrm{V}$ for $50 \leqslant T \leqslant 120 \mathrm{mK}$, and $5 \mu \mathrm{V}$ for $T>120 \mathrm{mK}$.

Due to their low mobilities our samples show broad quantum Hall plateaus with vanishing conductance $G=I / V$ around integer filling factors $\nu=n h / e B$. Near half integer filling factors the conductance is nonzero with a peak value of the order of $10 e^{2} / h$, corresponding to diagonal conductivities $\sigma_{x x}=(1 / 2 \pi) \ln (1+w / r) G \approx 0.15 e^{2} / h$, with an actual value between 0.1 and $0.3 e^{2} / h$ (depending on sample and filling factor). Here we concentrate on the plateau transition $\nu=2 \rightarrow 1$ which is broadest in magnetic field. The conductance at this transition is shown in Fig. 1(a) for different temperatures. It is dominated by the well understood conductance peak which arises when the Fermi energy sweeps through the delocalized electronic states near the center of a spin split Landau band.

Superimposed onto this conductance peak are small reproducible conductance fluctuations; see Fig. 1(b). For further analysis they first have to be separated from the peak form $\overline{G(B)}$ obtained by a polynomial smoothing. Only features on scales $\Delta B>50 \mathrm{mT}$ are included into $\overline{G(B)}$. The final fluctuations $\delta G(B)=G(B)-\overline{G(B)}$ extracted from the peak are shown in Fig. 1(c). Distinct reproducible features can be clearly observed, they become smoother with rising temperature and their amplitude shrinks.

Considering that the electronic transport in the $\mathrm{QH}$ plateau transition with extended states at the Fermi energy may be regarded as quasimetallic, it is worthwhile to compare the observed conductance fluctuations with UCF's in mesoscopic 2D metals, which are governed by the minimum of either the dephasing length, $L_{\phi}=\sqrt{D \tau_{\phi}}$, or the thermal 


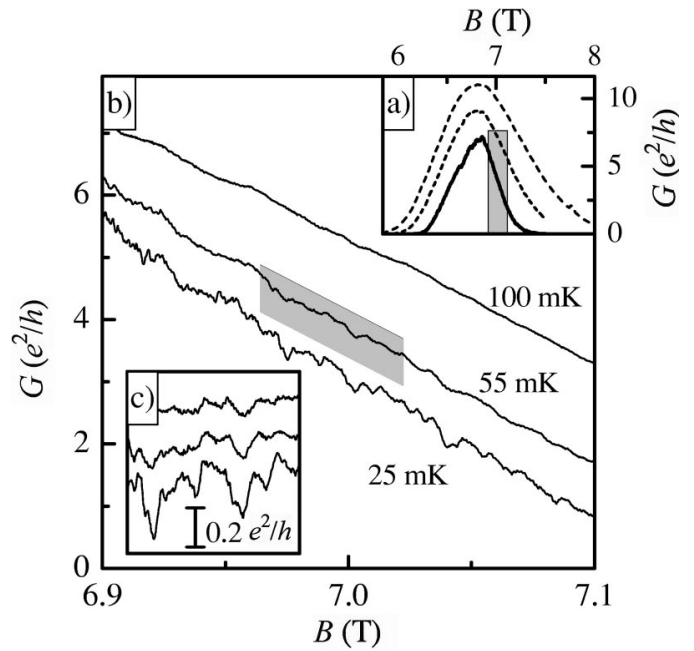

FIG. 1. a) Conductance peak at the $\nu=2 \rightarrow 1$ plateau transition for temperatures $T=55 \mathrm{mK}$ (solid line), $160 \mathrm{mK}$, and $320 \mathrm{mK}$. (b) Enlargement of the gray area in (a) for low temperatures where reproducible conductance fluctuations become visible. (c) Conductance fluctuations $\delta G(B)$ after subtraction of a smooth background $\overline{G(B)}$ for $B=6.97-7.02 \mathrm{~T}$ and the same temperatures as in (b). The scale of the plot is visualized by the gray area in (b). The curves are shifted for clarity.

length, $L_{T}=\sqrt{\hbar D / k_{B} T}{ }^{1}$ Here $D$ is the diffusion coefficient and $\tau_{\phi}$ is the phase coherence time.

For the low temperatures considered here $(T<1 \mathrm{~K})$ it was shown that in high magnetic fields electron-electron scattering dominates $\tau_{\phi}$ in a 2 DES realized in a semiconductor. ${ }^{13}$ For a typical conductivity $\sigma_{c} \sim 0.5 e^{2} / h$ near the critical point of the QH plateau transition it is estimated to $\tau_{\phi} \sim \hbar /\left(k_{B} T\right) .{ }^{14,15}$ In this framework the dephasing length $L_{\phi}$ approximately equals the thermal length $L_{T}$ and a distinction between $L_{\phi}$ and $L_{T}$ becomes unnecessary.

The dephasing length $L_{\phi}(T)$ influences the temperature dependence of both the amplitude of the conductance fluctuations, $\sqrt{\left\langle\delta G^{2}\right\rangle}$, and their correlations. This fact can be formalized when using a temperature-dependent correlation function averaged over the magnetic field, $F(\Delta B)$ $=\langle\delta G(B) \delta G(B+\Delta B)\rangle_{B}$. Defining a correlation field, $B_{c}(T) \approx \Phi_{0} / L_{\phi}(T)^{2}$, the correlation function then embraces two limits: ${ }^{1}$ It is constant for $\Delta B \ll B_{c}$, i.e., $F(\Delta B)=F(0)$ $=\left\langle\delta G^{2}\right\rangle$, reduced to $F\left(B_{c}\right)=F(0) / 2$ for $\Delta B=B_{c}$, and it follows $F(\Delta B) \propto 1 / \Delta B^{2}$ for $\Delta B \gg B_{c}$.

UCF's can be analyzed in terms of the power spectral density (PSD), defined as the Fourier transform of the correlation function $F(\Delta B)$,

$$
\mathcal{P}\left(f_{B}\right)=\frac{1}{2 \pi} \int F(\Delta B) \exp \left(-i 2 \pi f_{B} \Delta B\right) d \Delta B
$$

with $f_{B}$ the magnetic frequency. It has been shown ${ }^{16}$ that using the PSD yields a more accurate analysis of the data in an experimental situation like ours, where we are only able to measure fluctuations on a magnetic field scale $\Delta B$ $<0.05 \mathrm{~T}$, and, where we are additionally limited to a finite range of the magnetic field $(B=6.4-7.1 \mathrm{~T})$.
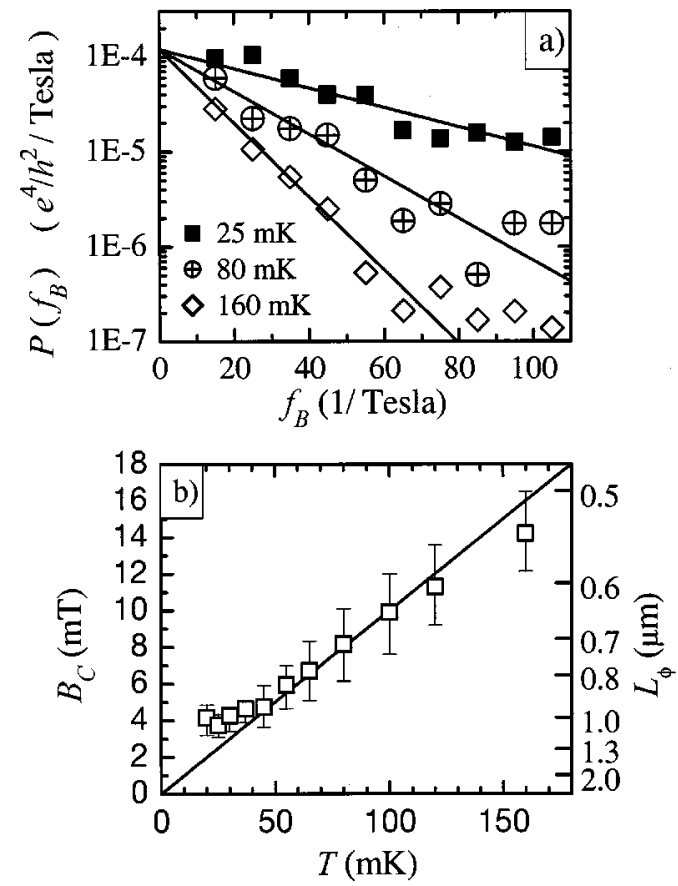

FIG. 2. (a) Power spectral density $\mathcal{P}\left(f_{B}\right)$ of the conductance fluctuation $\delta G(B)$ in a magnetic field interval $B=6.4-7.1$ T. The lines show fits to Eq. 2. (b) Correlation field $B_{c}$, respectively, dephasing length $L_{\phi}=\sqrt{\Phi_{0} / B_{c}}$ determined by the fits shown in (a). The straight line shows the expected linear dependence $B_{c} \propto T$, respectively, $L_{\phi} \propto 1 / \sqrt{T}$.

In Fig. 2(a) the PSD $\mathcal{P}\left(f_{B}\right)$ of our experimentally measured conductance fluctuations, $\delta G(B)=G(B)-\overline{G(B)}$, is shown for several temperatures. Each point within the graph is the result of averaging $\ln \left[\mathcal{P}\left(f_{B}\right)\right]$ over a frequency interval of $\Delta f_{B}=10 \mathrm{~T}^{-1}$. The data follow an exponential decay indicated by the straight lines. The observed saturation of the $T=160 \mathrm{mK}$ data for frequencies $f_{B} \geqslant 60 \mathrm{~T}^{-1}$ is caused by measurement noise which is constant with frequency and therefore always dominates the PSD at high $f_{B}$. For $T$ $>200 \mathrm{mK}$ the frequency interval, where the PSD does not sink into the measurement noise, becomes too small for a trustworthy analysis.

The exponential decay of the PSD observed in Fig. 2(a) can be fitted by a simple model,

$$
\mathcal{P}\left(f_{B}, B_{c}(T)\right)=\mathcal{P}_{0} e^{-2 \pi B_{c}(T) f_{B}} .
$$

Here $\mathcal{P}_{0}$ is a temperature-independent prefactor. The corresponding correlation function $F(\Delta B)$ fulfills all predictions for the limits of $F(\Delta B)$. Additionally, the temperature dependence of $\left\langle\delta G^{2}\right\rangle=F(0)$ extracted from this PSD follows the expected behavior for a 2D metal, $\left\langle\delta G^{2}\right\rangle \propto L_{\phi}^{2}(T)$ $\propto 1 / B_{c}(T) .{ }^{1}$ A least-square fit shown by the straight lines in Fig. 2(a) now enables us to determine the correlation field $B_{c}(T)$ plotted in Fig. 2(b). For $T \geqslant 50 \mathrm{mK}$ the correlation field follows a linear dependence $B_{c} \propto T$ as expected for $B_{c}$ $=\Phi_{0} / L_{\phi}^{2}$ and $L_{\phi} \propto T^{-1 / 2}$. The corresponding temperaturedependent dephasing length $L_{\phi}$ is shown on the right axis of Fig. 2(b). Down to a temperature $T=50 \mathrm{mK}$ it is still well 


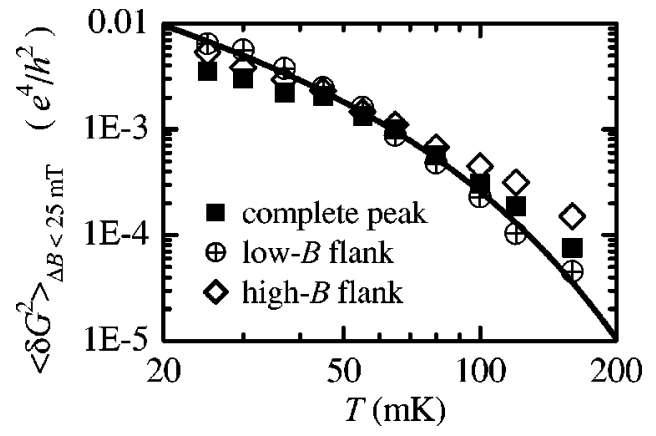

FIG. 3. Reduced amplitude of the conductance fluctuations as defined in Eq. (3) with $f_{g}=40 \mathrm{~T}^{-1}$. Data are shown for the complete magnetic field range $B=6.4-7.1 \mathrm{~T}$ used in Fig. 2, for the low- $B$ flank in an interval $B=6.4-6.6 \mathrm{~T}$, and the high- $B$ flank with $B=6.9-7.1$ T. The line shows the expectation from Eqs. (3) and (2) using $B_{c}(\mathrm{~T})=0.1 \cdot T(\mathrm{~K})$ linearly depending on $T$ as shown in Fig. 2(b).

below the width of the sample, $w=6 \mu \mathrm{m}$. The observed saturation of $L_{\phi}$ at temperatures below $T=50 \mathrm{mK}$ is presumably caused by a decoupling of the electron temperature from the bath temperature due to microwave heating.

Using the experimentally measured PSD we can now directly calculate the amplitude square $\left\langle\delta G^{2}\right\rangle$ of the UCF's. However, since our procedure of extracting $\delta G$ from the peak disregards fluctuations on a scale $\Delta B>50 \mathrm{mT}$ we have to restrict ourselves to frequencies $f_{B}$ above a cutoff frequency $f_{g}>(50 \mathrm{mT})^{-1}$. This defines a reduced fluctuation amplitude square,

$$
\left\langle\delta G^{2}\right\rangle_{\Delta B<\left(1 / f_{g}\right)}=2 \int_{f_{g}}^{\infty} d f_{B} \mathcal{P}\left(f_{B}\right),
$$

where mainly fluctuations on small magnetic field scales $\Delta B<1 / f_{g}$ are taken into account. For a large enough $f_{g}$ effects of the smooth background subtraction are negligible and we can safely extract an experimental UCF amplitude to be compared to theoretical calculations.

Figure 3 shows the measured reduced amplitude square of the fluctuations as defined in Eq. (3) for $f_{g}=40 \mathrm{~T}^{-1}$. The data are shown for the complete magnetic field interval $(6.4<B<7.1 \mathrm{~T})$ and for small $B$ intervals on both flanks of the peak around the points of half peak height $(6.4<B$ $<6.6 \mathrm{~T}$ and $6.9<B<7.1 \mathrm{~T}$ ). The experimental data are compared with the expectations from UCF theory, which using Eqs. (2) and (3) yields $\left\langle\delta G^{2}\right\rangle_{\triangle B<1 / f_{g}}=\mathcal{P}_{0} /\left(\pi B_{c}\right) \exp$ $\left(-2 \pi B_{c} f_{g}\right)$ with a critical field $B_{c}$ linearly depending on $T$. As shown in Fig. 3 the temperature dependence of all three data sets can be described by UCF theory.

Using this reduced amplitude as given in Eq. (3) or equivalent the experimentally determined PSD as given in Eq. (2) we can extrapolate from the raw experimental data to the full fluctuation amplitude $\sqrt{\left\langle\delta G^{2}(T)\right\rangle} \approx 0.02\left(e^{2} / h\right)$ $\times[1 / \sqrt{T(\mathrm{~K})}] \approx 0.1\left(e^{2} / h\right) L_{\phi}(\mu \mathrm{m})$. This value can be compared to the fluctuation amplitude

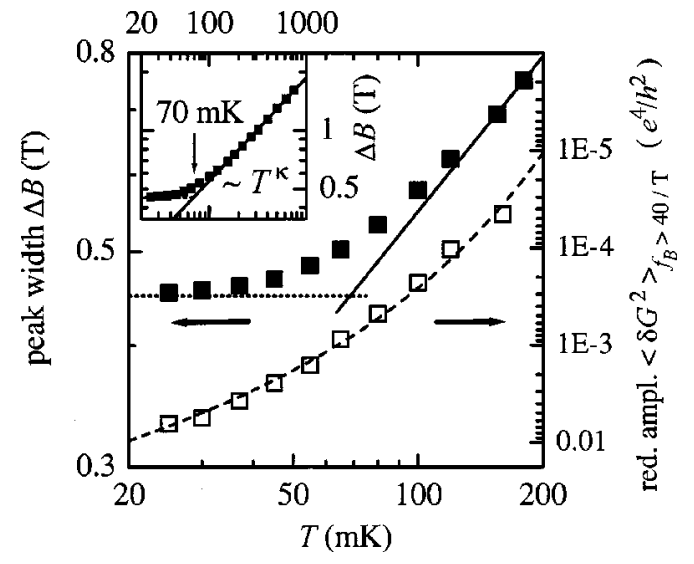

FIG. 4. The peak width ( $\mathbf{\square})$, measured as full width at half maximum $\Delta B$, shows scaling behavior $\Delta B \propto T^{\kappa}$ (straight line) with $\kappa=0.5$ at elevated temperatures (inset) and saturates due to finite size effects at low temperature (dotted line). A saturation of the electron temperature can be ruled out as the measured reduced fluctuation amplitude ( $\square$, low- $B$ flank from Fig. 3) follows the prediction [dashed line, $B_{c}(\mathrm{~T})=0.1 \cdot T(\mathrm{~K})$ ] down to $T \leqslant 30 \mathrm{mK}$.

$$
\sqrt{\left\langle\delta G^{2}(T)\right\rangle} \sim \frac{e^{2}}{h}\left(\frac{2 \pi r}{w}\right)^{1 / 2} \frac{L_{\phi}(T)}{w}
$$

for a ring-shaped metallic sample with radius $r$ and width $w .{ }^{1}$ An extension of UCF theory to the presence of Landau quantization does not predict any change in the universal fluctuation amplitude. ${ }^{17-20}$ With the parameters $w=6 \mu \mathrm{m}$ and $r$ $=60 \mu \mathrm{m}$ for our sample we expect $\sqrt{\left\langle\delta G^{2}\right\rangle}$ $\sim\left(e^{2} / h\right) L_{\phi}(\mu \mathrm{m})$, one order of magnitude larger than the experimental value deduced above. This discrepancy mirrors that the 2DES at the QH plateau transition can be described to some extend like a 2D metal but is not a metal. In fact, the conductivity $\sigma_{x x}<e^{2} / h$ does not allow the perturbative treatment to first order of $\left(e^{2} / h\right) / \sigma_{x x}$ used in UCF theory ${ }^{1,17}$ for metallic systems. However, numerical studies of the twopoint conductance $G$ of a rectangular sample at the transition from the Hall insulator $G=0$ to the lowest Hall plateau $G$ $=1$ with a Chalker and Coddington network model ${ }^{21,22}$ also observe a reduced amplitude $\sqrt{\left\langle\delta G^{2}(T)\right\rangle} \leqq 0.3 e^{2} / h$ compared to $\sqrt{\left\langle\delta G^{2}(T)\right\rangle} \sim e^{2} / h$ for high filling.

Finally, the temperature dependence of the fluctuations amplitude can be used to compare the length scale $L_{\phi} \sim L_{T}$ $\propto T^{-1 / 2}$ governing the fluctuations with the length $L_{s c}(T)$ $\propto T^{-1 / z}$ appearing in the scaling behavior of the $\mathrm{QH}$ plateau transition, $z$ is the so-called dynamical scaling exponent (for a review, see Ref. 23). For temperatures where the sample width $w$ exceeds $L_{s c}$ the transition width of the QH plateau transition scales as $\Delta B \propto L_{s c}^{-1 / \gamma} \propto T^{\kappa}$ with $\kappa=1 / z \gamma$. For lower temperatures $\Delta B$ was experimentally found to saturate. ${ }^{24}$ This saturation could be attributed to finite-size scaling occuring for $w<L_{s c}$ which allowed a direct measurement of the localization length in the $\mathrm{QH}$ plateau transition.

Usually $L_{s c}$ is identified as the dephasing length $L_{\phi}$. However, it was noted that for $L_{\phi}>w$ one would expect large mesoscopic fluctuations. ${ }^{25}$ In contrast, no fluctuations ${ }^{24}$ or only small fluctuations ${ }^{26}$ were observed experimentally at 
the lowest temperatures. As a consequence, the observed saturation of $\Delta B$ was claimed to be caused by other effects such as external heating of the 2 DES. ${ }^{25}$ In our experiments we can use the temperature dependence of the fluctuation amplitude as a thermometer for the 2DES. As can be seen from the temperature dependence of the correlation field in Fig. 2(b) and the fluctuation amplitude $\left\langle\delta G^{2}\right\rangle$ in Fig. 3 an electron temperature $T_{e}$ well below $50 \mathrm{mK}$ is achieved. For the lower conductance at the flanks, where unwanted microwave heating is reduced, $\left\langle\delta G^{2}\right\rangle$ even follows the model down $30 \mathrm{mK}$ (Figs. 3 and 4). In contrast, as shown in Fig. 4 the width $\Delta B$ of the conductance peak only follows a scaling law $\Delta \propto T^{\kappa}$ for $T \geqslant 150 \mathrm{mK}$ and saturates for $T<100 \mathrm{mK}$. Interpreted as a saturation decoupling of electron temperature from the temperature in the mixing chamber one would find a minimum electron temperature $T_{e}=70 \mathrm{mK}$ inconsistent with the observed temperature dependence of the fluctuations. This demonstrates that the observed transition width saturation is indeed an effect of the sample size. Additionally, using the generally accepted value $\gamma=2.3$ of the critical scaling exponent and applying the relation $\kappa=1 / z \gamma$ for the

*Electronic address: hohls@nano.uni-hannover.de

${ }^{1}$ P.A. Lee, A.D. Stone, and H. Fukuyama, Phys. Rev. B 35, 1039 (1987).

${ }^{2}$ G. Timp, A.M. Chang, P. Mankiewich, R. Behringer, J.E. Cunningham, T.Y. Chang, and R.E. Howard, Phys. Rev. Lett. 59, 732 (1987).

${ }^{3}$ A.K. Geim, P.C. Main, P.H. Beton, L. Eaves, S.P. Beaumont, and C.D.W. Wilkinson, Phys. Rev. Lett. 69, 1248 (1992).

${ }^{4}$ F. Hohls, U. Zeitler, and R.J. Haug, Ann. Phys. (N.Y.) 8, SI97 (1999).

${ }^{5}$ J.A. Simmons, S.W. Hwang, D.C. Tsui, H.P. Wei, L.W. Engel, and M. Shayegan, Phys. Rev. B 44, 12933 (1991).

${ }^{6}$ P.C. Main, A.K. Geim, H.A. Carmona, C.V. Brown, T.J. Foster, R. Taboryski, and P.E. Lindelof, Phys. Rev. B 50, 4450 (1994).

${ }^{7}$ A.A. Bykov, Z.D. Kvon, E.B. Ol'shanetskii, L.V. Litvin, and S.P. Moshchenko, Phys. Rev. B 54, 4464 (1996).

${ }^{8}$ D.H. Cobden and E. Kogan, Phys. Rev. B 54, R17 316 (1996).

${ }^{9}$ D.H. Cobden, C.H.W. Barnes, and C.J.B. Ford, Phys. Rev. Lett. 82, 4695 (1999).

${ }^{10}$ T. Machida, S. Ishizuka, S. Komiyama, K. Muraki, and Y. Hirayama, Phys. Rev. B 63, 045318 (2001).

${ }^{11}$ K. Ploog, J. Cryst. Growth 81, 304 (1987). critical exponents of the $\mathrm{QH}$ plateau transition one finds $L_{s c} \propto 1 / T .^{23}$ This deviates substantially from the temperature dependence of $L_{\phi} \propto 1 / \sqrt{T}$. Both observations demonstrate that size scaling and conductance fluctuations are governed by distinct length scales implying that $L_{s c}$ depends on different mechanisms than $L_{\phi}$, an experimental finding in agreement with recent theoretical predictions. ${ }^{15}$

In conclusion, we have measured the conductance fluctuations of a 2DES in the quantum Hall regime. Their dependence on temperature and magnetic field can be described by standard UCF theory for metals, however, their amplitude is considerably reduced. In addition we have shown that temperature dependence of the quantum Hall plateau transition and that of the fluctuations are governed by different length scales.

The samples used for our experiments were grown by K. Ploog at the Max Planck Institut für Festkörperforschung. We thank F. Evers, F. Kuchar, D. Polyakov, and L. Schweitzer for stimulating discussions. We acknowledge financial support from the DFG and DIP.

${ }^{12}$ R.J. Haug, R.R. Gerhardts, K.v. Klitzing, and K. Ploog, Phys. Rev. Lett. 59, 1349 (1987).

${ }^{13}$ T. Brandes, Phys. Rev. B 52, 8391 (1995).

${ }^{14}$ T. Brandes, L. Schweitzer, and B. Kramer, Phys. Rev. Lett. 72, 3582 (1994).

${ }^{15}$ D.G. Polyakov and K.V. Samokhin, Phys. Rev. Lett. 80, 1509 (1998).

${ }^{16}$ R. Schäfer, P. vom Stein, and C. Wallisser, in Advances in Solid State Physics, edited by B. Kramer (Vieweg, Braunschweig, 1999), Vol. 39, p. 583.

${ }^{17}$ S. Xiong and A.D. Stone, Phys. Rev. Lett. 68, 3757 (1992).

${ }^{18}$ D.L. Maslov and D. Loss, Phys. Rev. Lett. 71, 4222 (1993).

${ }^{19}$ D.E. Khmelnitskii and M. Yosefin, Surf. Sci. 305, 507 (1994).

${ }^{20}$ S. Xiong, N. Read, and A.D. Stone, Phys. Rev. B 56, 3982 (1997).

${ }^{21}$ S. Cho and M.P.A. Fisher, Phys. Rev. B 55, 1637 (1997).

${ }^{22}$ B. Jovanovic and Z. Wang, Phys. Rev. Lett. 81, 2767 (1998).

${ }^{23}$ B. Huckestein, Rev. Mod. Phys. 67, 357 (1995).

${ }^{24}$ S. Koch, R.J. Haug, K.v. Klitzing, and K. Ploog, Phys. Rev. Lett. 67, 883 (1991).

${ }^{25}$ D. Liu and S. Das Sarma, Phys. Rev. B 49, 2677 (1994).

${ }^{26}$ S. Koch, R.J. Haug, K.v. Klitzing, and K. Ploog, Phys. Rev. B 46, 1596 (1992). 